\title{
Excitations of the torelon
}

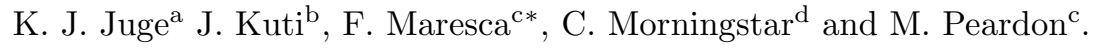

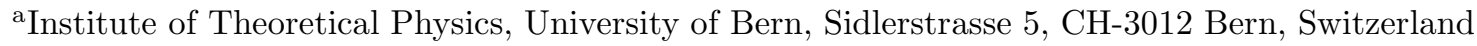 \\ ${ }^{b}$ Department of Physics, University of California at San Diego, La Jolla, USA 92093-0319 \\ 'School of Mathematics, Trinity College, Dublin 2, Ireland \\ ${ }^{\mathrm{d}}$ Department of Physics, Carnegie Mellon University, Pittsburgh, PA, USA 15213-3890
}

The excitations of gluonic flux tube in a periodic lattice are examined. Monte Carlo simulations from an anisotropic lattice are presented and the comparison with effective string models is discussed.

\section{INTRODUCTION}

It is believed that the confining regime of YangMills theory may be described by some kind of effective string model. The energy of the flux tube between static quark sources in $4 \mathrm{~d} \mathrm{SU}(3)$ has been studied in detail [12]: the grouping of excited states into bands and the corresponding energy gap dependence on $r$ at large distances is suggestive of the string picture, but there is a significant fine structure. Recent studies in $3 \mathrm{~d} \mathrm{Z}(2), \mathrm{SU}(2)$ and compact $\mathrm{U}(1)$ 3] confirmed a string formation at large separation with less pronounced fine structure. In the following calculations we analyse the spectrum of the QCD periodic flux tube. The torelon has no fixed colour sources and so provides a particularly favourable theoretical environment in which to observe the onset of string behaviour [4].

\section{SIMULATION DETAILS}

On the lattice, torelons may be created using colour-singlet traces of ordered product of link matrices encircling a spatial lattice direction. Excitations are obtained by projection onto longitudinal momentum $p_{z}$ eigenstates and onto different irreps of rotations in the plane transverse to the flux. The energy spectrum of a QCD flux tube is then estimated by Monte-Carlo measurement of

\footnotetext{
*Talk presented by F. Maresca
}

the correlation function,

$$
C(t)=\left\langle\phi^{\dagger}(t) \phi(0)\right\rangle \stackrel{t \rightarrow \infty}{\rightarrow}|\langle\operatorname{vac}|\phi| 0\rangle|^{2} e^{-E_{0} t},
$$

where $E_{0}$ is the energy of the lightest state which can be created by the operator $\phi(t)$. Because the signal decreases exponentially as $t$ is increased, it is very important to obtain the asymptotic behaviour of the correlator as quickly as possible. In order to do so we choose operators for which the overlap with the lightest state is as large as possible. We use an anisotropic lattice in which the temporal spacing $a_{t}$ is much smaller than that in the spatial directions $a_{s}$ to exploit the enhanced signal-to-noise of the correlation function at smaller temporal separations. The center symmetry of the Lagrangian allows us to restrict our torelon operators to be positive under charge conjugation. The lattice symmetry of rotations about the winding axis $z$, is $C_{4 \nu} \otimes Z(\mathcal{R})$ for $p_{z}=0$ or $C_{4 \nu}$ for $p_{z} \neq 0 . C_{4 \nu}$ contains rotations of $\frac{\pi}{2}$ and the reflection in the $x y$ plane ( $\mathcal{P}$-parity) and has five irreducible representations: $A_{1}, A_{2}, B_{1}, B_{2}$ and E. $Z(\mathcal{R})$ denotes the two-element group consisting of the identity operation and the reflection about the midpoint on the principal axis $z(\mathcal{R}$ parity). States that are even/odd under $\mathcal{R}$-parity are labeled by the subscripts $g / u$ respectively.

Simulations were performed on three different lattices of extent in the $z$ direction of 8,12 and $16 a_{s}$. The lattice action was Symanzik improved and included a two-plaquette term, designed to reduce cut-off artefacts in the scalar glueball 


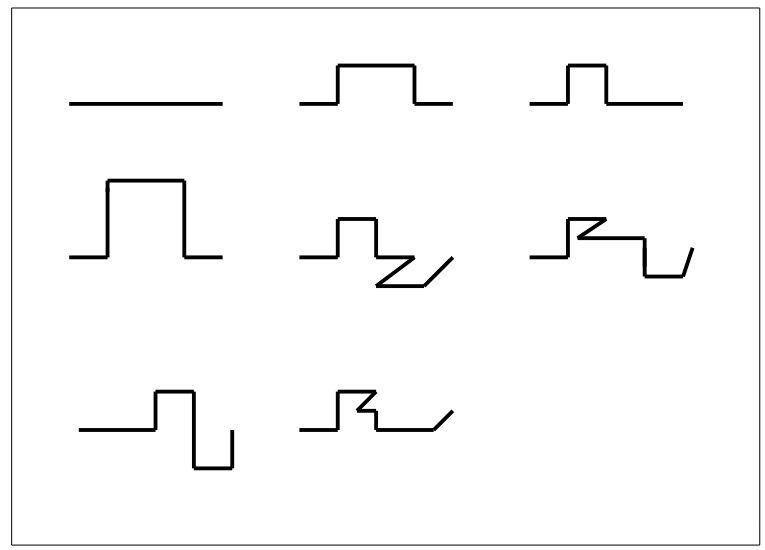

Figure 1. The eight prototype lattice paths used in the construction of torelon operators in this work.

mass. The anisotropy was set to $a_{s} / a_{t}=6$ with $a_{s}=0.21 \mathrm{fm}$ (from $r_{0}$ ). Linear combinations of the eight operators shown in Fig. 1 transforming irreducibly under the lattice symmetries were computed on two smeared sets of links and a variational methods was employed on the 16 operators to optimise overlaps.

\section{EFFECTIVE STRING THEORY}

For long QCD torelons, the two transverse oscillations of the tube are the only degrees of freedom forced to be low-lying Goldstone modes. These are the degrees of freedom of an oscillating string. In $d<26$ however, quantisation spoils Lorentz invariance and the string picture must then be regarded as an effective theory.

An important question is whether or not sufficiently long QCD torelons can be well described by an effective string theory, such as the Nambu-Goto string in $d<26$, or the PolchinskiStrominger proposal 5. Note that the simplest theories do not naturally include a number of effects that might also be relevant, such as rigidity terms or interactions with the QCD bulk.

At lowest order in an expansion about $1 / L$, all these pictures give a common spectrum of states with energy gaps $2 \pi N / L$. The quantum numbers of some of these excitations for states up to $N=3$ are given in Table 1 This table is restricted to
Table 1

Lowest string energy levels and their corresponding string and QCD states. The operator $a_{m}^{s(d) \dagger}$ creates an $\mathrm{m}$ string mode of right(left) chirality.

\begin{tabular}{lll}
\hline Level & State & QCD $L_{R}^{P}\left(p_{z}\right)$ \\
\hline 0 & $|0\rangle$ & $\Sigma_{g}^{+}(0)$ \\
\hline 1 & $\left(a_{1}^{s \dagger}+a_{1}^{d \dagger}, a_{1}^{s \dagger}-a_{1}^{d \dagger}\right)|0\rangle$ & $\Pi(1)$ \\
\hline 2 & $\left(a_{1}^{s \dagger} \tilde{a}_{1}^{s \dagger}+a_{1}^{d \dagger} \tilde{a}_{1}^{d \dagger}\right)|0\rangle$ & $\Delta_{g}(0)$ \\
& $\left(a_{1}^{s \dagger} \tilde{a}_{1}^{s \dagger}-a_{1}^{d \dagger} \tilde{a}_{1}^{d \dagger}\right)|0\rangle$ & $\Delta_{g}(0)$ \\
& $\left(a_{1}^{d \dagger} \tilde{a}_{1}^{s \dagger}-a_{1}^{s \dagger} \tilde{a}_{1}^{d \dagger}\right)|0\rangle$ & $\Sigma_{u}^{-}(0)$ \\
& $\left(a_{1}^{d \dagger} \tilde{a}_{1}^{s \dagger}+a_{1}^{s \dagger} \tilde{a}_{1}^{d \dagger}\right)|0\rangle$ & $\Sigma_{g}^{+*}(0)$ \\
\cline { 2 - 3 } & $\left(\left(a_{1}^{s \dagger}\right)^{2}+\left(a_{1}^{d \dagger}\right)^{2}\right)|0\rangle$ & $\Delta(2)$ \\
& $\left(\left(a_{1}^{s \dagger}\right)^{2}-\left(a_{1}^{d \dagger}\right)^{2}\right)|0\rangle$ & $\Delta(2)$ \\
& $a_{1}^{d \dagger} a_{1}^{s \dagger}|0\rangle$ & \\
& $\left(a_{2}^{s \dagger}+a_{2}^{d \dagger}, a_{2}^{s \dagger}-a_{2}^{d \dagger}\right)|0\rangle$ & $\Pi(2)$ \\
\hline $3^{1}$ & $\left(\tilde{a}_{1}^{s \dagger} a_{2}^{s \dagger}+\tilde{a}_{1}^{d \dagger} a_{2}^{d \dagger}\right)|0\rangle$ & $\Delta(1)$ \\
& $\left(\tilde{a}_{1}^{s \dagger} a_{2}^{s \dagger}-\tilde{a}_{1}^{d \dagger} a_{2}^{d \dagger}\right)|0\rangle$ & $\Delta(1)$ \\
& $\left(\tilde{a}_{1}^{d \dagger} a_{2}^{s \dagger}+\tilde{a}_{1}^{s \dagger} a_{2}^{d \dagger}\right)|0\rangle$ & $\Sigma^{+}(1)$ \\
\hline
\end{tabular}

${ }^{1}$ This level includes also the states $0^{-}$and $1^{\star}$ with $\mathrm{p}=1$ and states with $\mathrm{p}=3$ not considered in our calculations

those states considered in the QCD calculation. This spectrum can be computed for the NambuGoto action and a computation of the $1 / L^{3}$ corrections in the Polchinski-Strominger scheme is also under investigation. The picture emerging from the analysis of these simple classes of effective string theories is that the fine structure is predominantly dependent on the longitudinal momentum along the string.

\section{PRELIMINARY RESULTS}

The results from the three simulations described in Sec. 2 are presented in Fig. 2 The value $L=2 \mathrm{fm}$ (determined from $r_{0}$ ) is indicated by a vertical line. As can be seen in the first figure, all states are correctly ordered according to their string classification, $N$ once $L>2 \mathrm{fm}$. The energy gap of all levels is qualitatively in agreement with the first order string prediction $2 \pi N / L$ as shown in the last figure.

The second figure shows the $N=1$ level is close to the lowest-order string prediction, although deviations are resolved. The difference between the lowest-order string model and this excitation ap- 
pears to grow as $\mathrm{L}$ is increased.

In the $N=2$ states, significant structure is seen below $2 \mathrm{fm}$, while an extremely interesting pattern of degeneracies emerges at $L / a_{s}=12$ and 16. Here, the $p_{z}=0$ and $p_{z}=2$ states form two degenerate clusters. This is in agreement with indications from the effective string calculations that the fine structure is dependent on $p_{z}$ alone. The splitting between these clusters decreases as $L$ increases.

At $N=3$, for $L>2 \mathrm{fm}$, there appears to be a single degenerate set (notice from Table. 11 all 3 states have $p_{z}=1$ ) but a clear split between the $B_{1}$ and $B_{2}$ states is found. In the continuum these two states are expected to be degenerate and tests on possible lattice artifacts are under investigation. Extra operators for these states are being studied.

\section{CONCLUSIONS}

In this work, we have demonstrated that highresolution measurements of the QCD torelon and its excitations can be made on the anisotropic lattice. This data will provide a useful forum for making comparisons with effective string theories and other models of the confining flux. Qualitative predictions of this fine structure remains a significant challenge. Continuum extrapolations are needed and simulations with larger torelons are also under study.

This work was supported by the U.S. NSF under Award PHY-0099450, U.S. DOE Grant No. DE-FG03-97ER40546, EU HPRN-CT-200000145. FM is grateful for support from Trinity College and Enterprise-Ireland.

\section{REFERENCES}

1. J.K. Juge, J. Kuti, C. Morningstar, Phys.Rev.Lett.90:161601,2003.

2. J.K. Juge, J. Kuti, C. Morningstar, Nucl.Phys.Proc.Suppl.106:691-693,2002

3. C. Morningstar and J. Kuti, Confinement 2003, RIKEN.

4. B. Lucini and M. Teper, Phys. Rev.D64:105019,2001.

5. J. Polchinski and A. Strominger, Phys.Rev. Lett.67:13,1991.
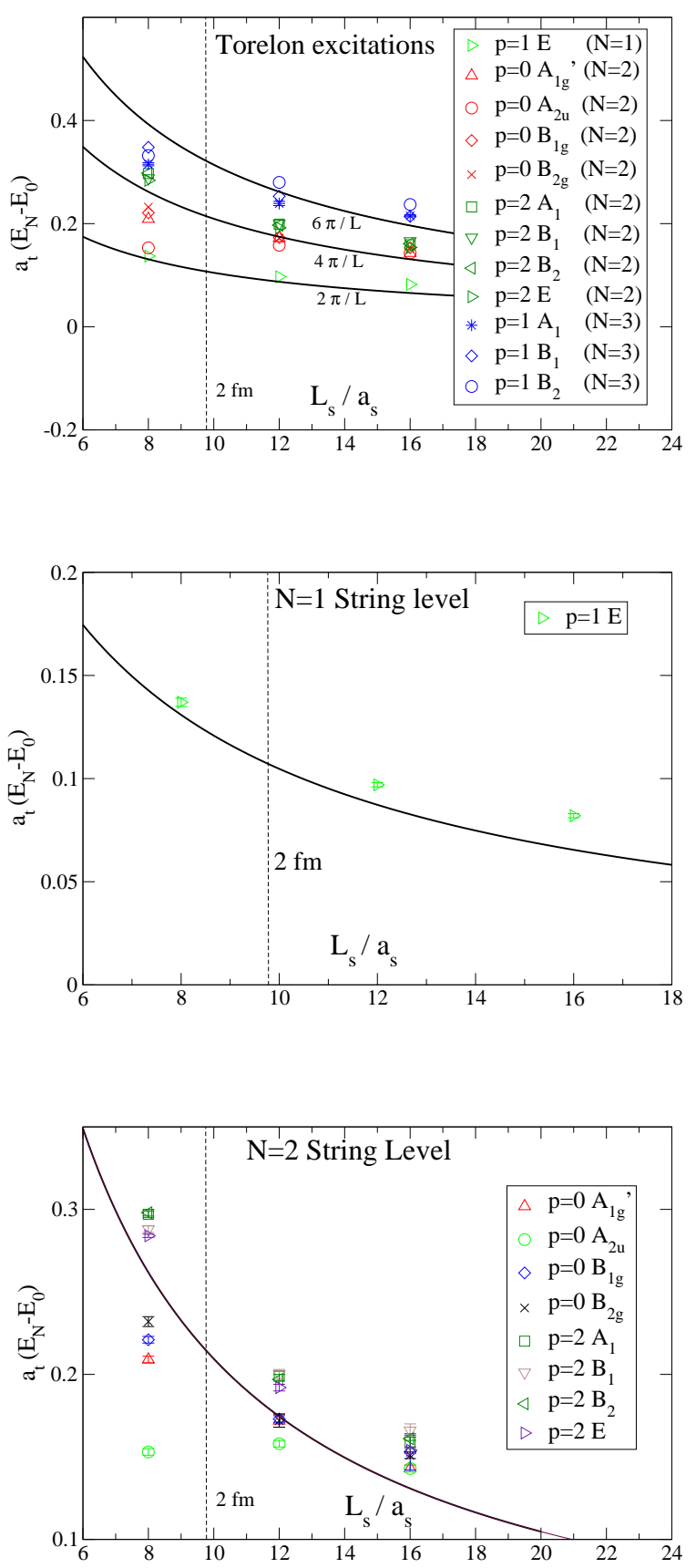

Figure 2. Energies of torelons of different lengths. The solid lines are the first order string prediction, $2 \pi N / L$. 